\title{
Footwear alterations after first metatarsophalangeal joint arthrodesis
}

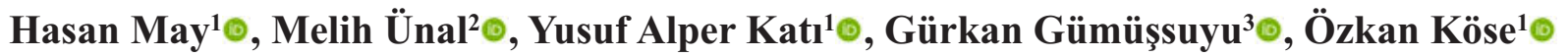

${ }^{1}$ Department of Orthopedics and Traumatology, Antalya Training and Research Hospital, Antalya, Turkey

${ }^{2}$ Department of Orthopedics and Traumatology, Yatağan State Hospital, Muğla, Turkey

${ }^{3}$ Department of Orthopedics and Traumatology, Altınbaş University, Medical Faculty, İstanbul, Turkey

\begin{abstract}
Objectives: This study aimed to examine the footwear alterations following first metatarsophalangeal (MTP1) joint arthrodesis.

Methods: A retrospective review was performed on 37 (42 feet) patients who underwent MTP1 joint arthrodesis between January 2014 and January 2019 in our institution. Functional outcomes were assessed with the American Orthopedic Foot and Ankle Society Hallux metatarsophalangeal-interphalangeal (AOFAS-MTPIP) scale, and the pain was assessed using the visual analog scale (VAS). Besides, time to return regular footwear, shoe preferences, the cosmetic appearance of the feet were evaluated and compared with preoperative status.
\end{abstract}

Results: The mean follow-up time was $12.0 \pm 16.5$ months. The preoperative AOFAS-MTP-IP scale improved from $46.6 \pm 7.0$ points to $86.2 \pm 7.2$ points $(p=0.001)$, and VAS decreased from $6.3 \pm 1.8$ points to $1.4 \pm 0.5$ points $(p=0.001)$. There was non-union in three $(7.1 \%)$ feet, and the mean union time for the rest of the feet was $8.2 \pm 1.1$ weeks. No other complications were seen. The mean time for resuming regular footwear was $11.8 \pm 2.6$ weeks. Patients' perception of the appearance of their feet improved from $3.9 \pm 2.8$ to $9.0 \pm 0.7$ points $(p=0.001)$. Twenty-nine $(78.6 \%)$ patients $(33 \mathrm{feet})$ reported that there was no restriction in footwear compared to the past, but $8(21.4 \%)$ patients had to choose specific types and models. None of the patients had to use modified or custom-made shoes. There was significant change in the heel height preferences after the surgery $(p=0.004) .18(52.4 \%)$ patients had to choose shorter heel height than preoperative preferences, whereas $19(47.6 \%)$ patients' preference did not change. Almost all patients, $36(97.6 \%)$ were using athletic shoes in their routine daily life.

Conclusions: MTP1 joint arthrodesis is a reliable method that controls pain and increases function with a low rate of complications. However, significant alterations in footwear preferences may occur. Patients should be informed adequately about these outcomes to modify patient expectations.

Keywords: Hallux valgus, hallux rigidus, arthrodesis, footwear, outcome

Fisula irst metatarsophalangeal (MTP1) joint arthrodesis is usually indicated in the end-stage osteoarthritis of the MTP1 joint that is refractory to conservative management, severe hallux valgus deformity more than $40^{\circ}$ of hallux valgus angle (HVA), and salvage procedure after unsuccessful primary forefoot surgery $[1,2]$. This procedure is an effective solution that controls pain and provides deformity correction with a 
low rate of complications [3]. However, MTP1 joint arthrodesis is a joint destructive procedure that completely restricts motion and eliminates the normal functions of the MTP1 joint. Consequently, gait and footwear alterations may occur $[4,5]$. MTP1 joint plays a vital role in walking. At least 60 degrees of extension is required starting from heel-off to toe-off during a normal gait cycle. Moreover, it must be stable enough to carry the body weight and act as a lever for forward propulsion [6].

Previous studies on gait analysis following MTP1 joint arthrodesis have shown significant changes such as decreased power of push-off, cadence and decreased step length, deterioration in plantar pressure distribution, and load to lesser toes, and compensation of hindfoot and forefoot movements to restore normal gait [3-7]. All these adverse changes in the gait kinematics and foot biomechanics also affect footwear preferences and use. There are quite a limited number of studies examining how footwear has been altered after this procedure in the current literature $[8,9]$. However, when informing patients about this surgery and obtaining consent, one of the questions frequently asked by patients is footwear changes [9]. Furthermore, footwear alterations have been shown to be closely associated with patient satisfaction in forefoot surgery [10].

The aim of this study is to examine the functional results after the MTP1 joint arthrodesis by focusing on the alterations of footwear.

\section{METHODS}

\section{Study Design and Patients}

A retrospective review was performed on digital medical records to identify all patients who underwent the first metatarsophalangeal joint (MTPJ) arthrodesis between January 2014 and January 2019 in our institution. The institutional patient database was used to collect all radiological imaging records in the Picture Archiving and Communication System (PACS), patient charts, operation reports, medical records, and the notes taken during follow-up visits. Patients with incomplete medical records, imaging files, and patients with less than one-year follow-up were excluded from the study.

During the time interval under study, a total of 52 patients were identified who underwent MTPJ arthrodesis. Of these, ten patients were lost in followup, three patients declined to participate, and two patients had improper medical and radiological records. Thus, 37 patients (42 feet) with a mean age of $58.6 \pm$ 13.7 years (range, 20-74 years) were included in the final analysis. Indication of arthrodesis was hallux rigidus in 14 feet and hallux valgus in 28 feet. This research was performed in compliance with the ethical principles set out in the 1964 Helsinki Declaration and its later modifications, and the study protocol was approved by the institutional review board (Approval date and issue: 2020/033).

\section{Surgical Technique and Postoperative Follow-up}

Patients were operated on under spinal anesthesia and tourniquet control. A dorsomedial incision was made, and the superficial cutaneous nerve was dissected and protected. After capsulotomy, all osteophytes were removed; in hallux valgus cases, a bunionectomy was also performed if necessary. Removal of cartilage was performed down to subchondral cancellous bone using cup and cone reamers. The MTP1 joint was held in the desired arthrodesis position with a contemporary $\mathrm{K}$ wires fixation, and fluoroscopic control was made before definitive implantation. A dorsal MTP arthrodesis locking plate was used for the fixation, and additional interfragmentary screw fixation was used in 14 feet (Fig. 1). A short-leg plaster cast was applied to all the patients for

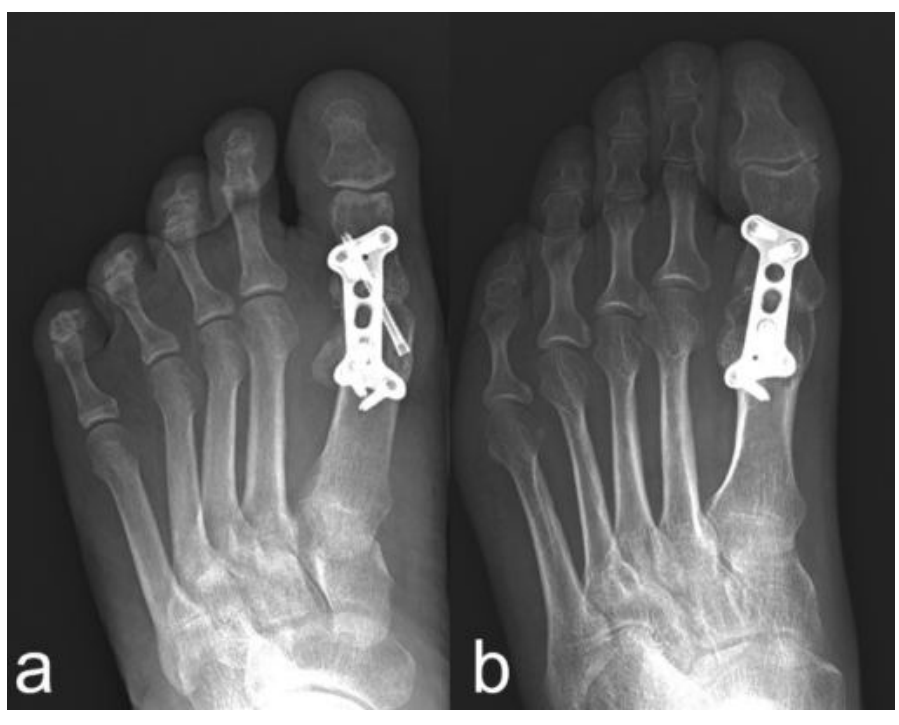

Fig. 1. (a) Dorsal plate with interfragmentary screw fixation (b) Single dorsal plate. 
the first two weeks. At the end of the second week, the cast was removed, and the patient was encouraged weight-bearing as tolerated on the heel and lateral side of the foot while the patient was wearing stiff-soled shoes. After the detection of the radiographic and clinical bony union at follow-up, full weight-bearing with regular shoes was allowed.

\section{Radiographic Evaluations}

Hallux valgus angle (HVA) and the first and second intermetatarsal angle (IMA) were measured on preoperative and final follow-up radiographs. Measurements were taken according to the recommendations of the ad hoc committee of the American Orthopedic Foot and Ankle Society [11]. Coughlin and Shurnas classification was used for grading of hallux rigidus cases [12]. Union of arthrodesis was evaluated on serial follow-up radiographs, and bridging bone formation on at least three cortex and arthrodesis site was accepted as a union.

\section{Clinical Evaluations}

Functional outcomes were assessed with the American Orthopedic Foot and Ankle Society Hallux metatarsophalangeal-interphalangeal (AOFAS-MTP-
IP) scale, and the pain was assessed using the visual analog scale (VAS) [13]. Preoperative and final follow-up scores were compared. In addition, a questionnaire was prepared to assess the effect of arthrodesis on footwear alterations (Table 1).

\section{Statistical Analysis}

Statistical analysis was performed using SPSS Statistics Base v.23 for Windows. Continuous variables were presented as mean \pm standard deviation, median, and range. Categorical variables were stated as percentages and frequency distribution. The Kolmogorov-Smirnov test was used to determine whether the data were distributed normally. Comparative analysis of dependent variables was performed using the Chi-square test for categorical variables, Wilcoxon Signed rank test for continuous variables in accordance with the normality testing. A value of $p<0.05$ was accepted as statistically significant.

\section{RESULTS}

There were 37 patients ( 9 male, 28 female) with a mean age of $58.6 \pm 13.7$ years (range, 20-74 years).

\section{Table 1. The questionnaire that was used for the assessment of footwear alteration after MTP1 joint arthrodesis}

1. How many weeks did it take to start wearing regular footwear after the operation?

2. What was your heel size preference before the operation?
a. Up to $2 \mathrm{~cm}$
b. Up to $4 \mathrm{~cm}$
c. Up to $6 \mathrm{~cm}$
d. More than $7 \mathrm{~cm}$

3. Currently, what heel size do you prefer for your comfort?
a. Up to $2 \mathrm{~cm}$
b. Up to $4 \mathrm{~cm}$
c. Up to $6 \mathrm{~cm}$
d. More than $7 \mathrm{~cm}$

4. Currently, could you choose your footwear without restriction?

$\begin{array}{ccc}\begin{array}{c}\text { a. I can tolerate any kind } \\ \text { of shoe without } \\ \text { restriction. }\end{array} & \begin{array}{c}\text { b. I can tolerate only } \\ \text { comfortable shoes; my } \\ \text { footwear selection is partly } \\ \text { restricted. }\end{array} & \begin{array}{c}\text { c. I cannot tolerate regular shoes, and I have to } \\ \text { choose specific brands with high toe box shoes. } \\ \text { My footwear selection is severely restricted. }\end{array}\end{array}$

5. Which shoe type do you prefer in your daily life?
a. Formal shoes
b. Casual shoes
c. Athletic shoes

6. How would you score the appearance of your feet preoperatively on a scale 1-10, 1 being the poor and 10 being perfect?

$\begin{array}{rrrrrrrrrr}1 & 2 & 3 & 4 & 5 & 6 & 7 & 8 & 9 & 10\end{array}$

7. How would you score the appearance of your feet postoperatively on a scale of 1-10, 1 being the poor and 10 being perfect?
1
2
3
4
5
6
7
8
9
10 
Five patients had bilateral MTPJ arthrodesis; thus, 42 feet (24 right, 18 left) were analyzed. Preoperative diagnosis was hallux rigidus in 14 feet 4 Grade III and 10 Grade IV) and hallux valgus in 28 feet (HVA $>40^{\circ}$ for all). A dorsal plate was used in 14 feet, and a dorsal plate with an additional interfragmentary compression screw was used in 28 feet.

The mean follow-up time was $12.0 \pm 16.5$ months (range, 12-72 months). The preoperative AOFASMTP-IP scale improved from $46.6 \pm 7.0$ points to 86.2 \pm 7.2 points $(p=0.001)$, and VAS decreased from 6.3 \pm 1.8 points to $1.4 \pm 0.5$ points $(p=0.001)$. There was non-union in three $(7.1 \%)$ feet, and the mean union time for the rest of the feet was $8.2 \pm 1.1$ weeks (range, 6-12 weeks) (Fig. 2). No other complications were seen. Computerized tomography was performed in non-union cases, and it revealed fibrous type nonunion in two cases without loss of position, and there was a loss of position in one patient with failure of implants (Fig. 3). Although revision surgery was offered, all denied a secondary intervention.

The mean time for resuming regular footwear was $11.8 \pm 2.6$ weeks (range, 8 -20 weeks). Patients' perception on the appearance of their feet improved from $3.9 \pm 2.8$ (range, $1-8$ ) to $9.0 \pm 0.7$ (range, $8-10$ ) points $(p=0.001)$. Twenty-nine $(78.6 \%)$ patients (33 feet) reported that there was no restriction in the selection of footwear compared to the past, but $8(21.4 \%)$ patients ( 9 feet) had to choose specific types and models.

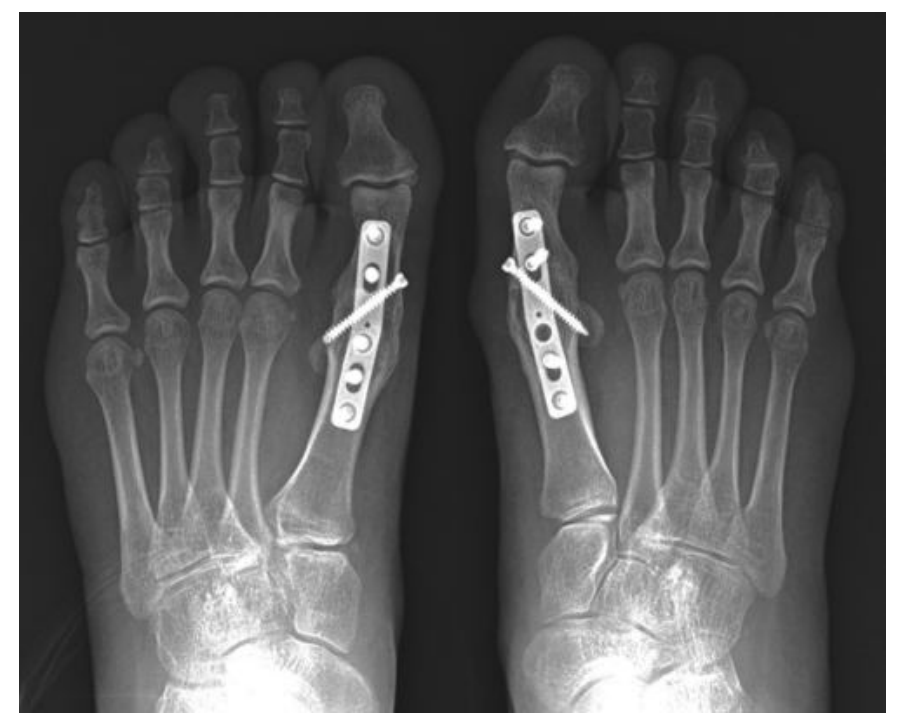

Fig. 2. Radiographic results of a patients with dorsal plate ad interfragmentary screw fixation at the final followed. Complete bony consolidation was seen.
None of the patients had to use modified or custommade shoes with complete restriction. There was a significant change in the heel height preferences after the surgery $(p=0.004)$ (Table 2$)$. Eighteen (22 feet, $52.4 \%$ ) patients had to choose shorter heel height compared to preoperative preferences, whereas 19 patients' (20 feet, $47.6 \%$ ) preference did not change. Almost all patients, 36 (41 feet, 97.6\%) were using athletic shoes in their daily life.

\section{DISCUSSION}

The current study examined the functional outcomes and footwear alterations after the MTP1 joint arthrodesis. Results of this study showed that functional outcomes were significantly improved compared to preoperative period and the pain was adequately decreased. Subjective rating on the appearance of the foot was also improved. No major complication was seen except nonunion in three feet. In this respect, MTP1 joint arthrodesis is a safe and reliable treatment for end-stage hallux rigidus and severe hallux valgus cases.

However, half of the patients switched to wear shoes with less heel height than they used to. In the other half, there was no change in heel selection, but none of the patients could wear high-heel shoes (over $7 \mathrm{~cm}$ ) before surgery. One out of five patients reported

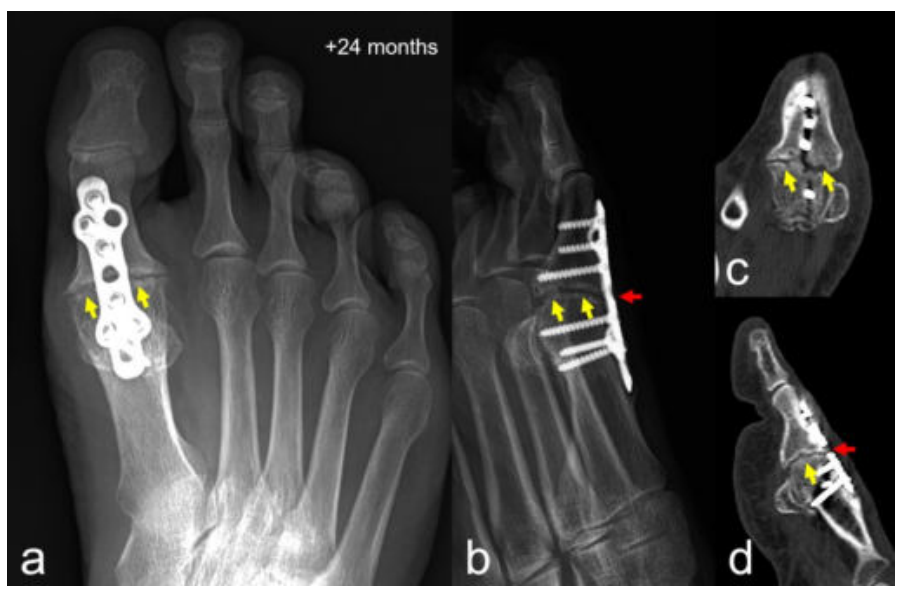

Fig. 3. Anteroposterior (a), and lateral (b) foot radiographs showing nonunion (yellow arrows) and failure of the dorsal plate (red arrow). Axial (c) and sagittal (d) computerized tomography demonstrate fibrous nonunion (yellow arrows) and discontinuity of the dorsal plate (red arrow). 
Table 2. Summary of results

\begin{tabular}{lccc}
\hline Variable & Preoperative & Postopoperative & $p$-value \\
\hline AOFAS (points \pm SD) & $46.6 \pm 7.0$ & $86.2 \pm 7.2$ & $\mathbf{0 . 0 0 1}$ \\
VAS (points \pm SD) & $6.3 \pm 1.8$ & $1.4 \pm 0.5$ & $\mathbf{0 . 0 0 1}$ \\
Appearance of Feet (points \pm SD) & $3.9 \pm 2.8$ & $9.0 \pm 0.7$ & $\mathbf{0 . 0 0 1}$ \\
Heel Size Preference (n) & & & $\mathbf{0 . 0 0 4}$ \\
$\quad$ Up to $2 \mathrm{~cm}$ & 9 & 22 & \\
Up to 4cm & 23 & 0 & \\
Up to 6cm & 10 & & \\
\hline
\end{tabular}

that they had to prefer certain shoe models for their comfort. Almost all patients reported to choose athletic shoe models in their routine daily life. In the light of these findings, it can be said that MTP1 joint arthrosis significantly alters the footwear preferences of the patients.

Shoes have emerged as a protective garment that facilitates walking in the outdoor environment and reduces injury, but has become a social theme. It is one of the most important components of fashion. It is also an indicator of socio-cultural status. Therefore, freedom in footwear preference is a factor that increases quality of life. In a recent study that conducted on large number of patients who underwent forefoot surgery, wearable range of shoes was reported as the one of the three factors that influence the final patient satisfaction [10]. DeSandis et al. [8] reported that 79\% of the patients were limited to wearing comfort shoes only following MTP1 joint arthrodesis. Our findings are consistent with these results. Consequently, it is necessary to provide detailed information on the footwear alterations following this procedure at the time of consultation. Alternative treatment methods should be considered in patients with high expectations or those who do not accept these alterations. Osteotomies that preserve joint movements, resection or interposition arthroplasty, and implant arthroplasty might be better option for these patients.

\section{Limitations}

The most obvious shortcoming of this study was retrospective data collection on small group of patients. Secondly, both hallux valgus and hallux rigidus cases were included although same procedure was per formed to all patients.

\section{CONCLUSION}

In conclusion, MTP1 arthrodesis is a reliable solution end-stage hallux rigidus and selected hallux valgus cases. However, significant footwear alterations might occur, such as wearing flat and athletic shoes. Surgeons should explain these issues in detail to modify patient expectations.

\section{Authors' Contribution}

Study Conception: HM, MÜ; Study Design: GG, ÖK; Supervision: ÖK; Funding: HM; Materials: HM; Data Collection and/or Processing: HM, YAK; Statistical Analysis and/or Data Interpretation: MÜ; Literature Review: MÜ, GG; Manuscript Preparation: HM and Critical Review: YAK, ÖK.

\section{Conflict of interest}

The authors disclosed no conflict of interest during the preparation or publication of this manuscript.

\section{Financing}

The authors disclosed that they did not receive any grant during conduction or writing of this study.

\section{REFERENCES}

1. Fuhrmann RA. First metatarsophalangeal arthrodesis for hallux rigidus. Foot Ankle Clin 2011;16:1-12.

2. Wood EV, Walker CR, Hennessy MS. First metatarsophalangeal arthrodesis for hallux valgus. Foot Ankle Clin 2014; 19:245-58.

3. Stevens J, de Bot RTAL, Hermus JPS, van Rhijn LW, Witlox 
AM. Clinical outcome following total joint replacement and arthrodesis for hallux rigidus: a systematic review. JBJS Rev 2017;5:e2.

4. DeFrino PF, Brodsky JW, Pollo FE, Crenshaw SJ, Beischer AD. First metatarsophalangeal arthrodesis: a clinical, pedobarographic and gait analysis study. Foot Ankle Int 2002;23:496-502. 5. Van Gheluwe B, Dananberg HJ, Hagman F, Vanstaen K. Effects of hallux limitus on plantar foot pressure and foot kinematics during walking. J Am Podiatr Med Assoc 2006;96:428-36. 6. Boissonnault WG, Donatelli RA. The influence of hallux extension on the foot during ambulation. J Orthop Sports Phys Ther 1984;5:240-2.

7. Stevens J, Meijer K, Bijnens W, Fuchs MC, van Rhijn LW, Hermus JP, et al. Gait analysis of foot compensation after arthrodesis of the first metatarsophalangeal joint. Foot Ankle Int 2017;38:181-91.

8. DeSandis B, Pino A, Levine DS, Roberts M, Deland J, O'Malley M, et al. Functional outcomes following first metatarsophalangeal arthrodesis. Foot Ankle Int 2016;37:715-21.

9. Rajczy RM, McDonald PR, Shapiro HS, Boc SF. First metatar- sophalangeal joint arthrodesis. Clin Podiatr Med Surg 2012;29:41-9.

10. Dawson J, Boller I, Doll H, Lavis G, Sharp RJ, Cooke P, et al. Factors associated with patient satisfaction with foot and ankle surgery in a large prospective study. Foot (Edinb). 2012;22:2118.

11. Coughlin MJ, Saltzman CL, Nunley 2nd JA. Angular measurements in the evaluation of hallux valgus deformities: a report of the ad hoc committee of the American Orthopaedic Foot and Ankle Society on angular measurements. Foot Ankle Int 2002;23:68-74.

12. Coughlin MJ, Shurnas PS. Hallux rigidus: demographics, etiology and radiographic assessment. Foot Ankle Int 2003;24:73143.

13. Kitaoka HB, Alexander IJ, Adelaar RS, Nunley JA, Myerson MS, Sanders M. Clinical rating systems for the ankle-hindfoot, midfoot, hallux, and lesser toes. Foot Ankle Int 1994;15:349-53. 